\title{
Características que influyen al error humano en la toma de decisiones estratégicas de los niveles directivos en la Industria Siderúrgica Mexicana (Characteristics that influence human error in the strategic decision-making at the top levels of the Mexican steel industry)
}

\section{'Fernando Monroy Guajardo iiJoel Mendoza Gómez}

\begin{abstract}
The strategic decision-making process is a keystone for companies to maintain their competitive advantage and prevail in the future. The purpose of this work was to identify the human error factors in the strategic decision-making process that influence bias at executive levels of the Mexican steel industry. Within the factors were, among others, emotionality, cognitive complexity, decision timing, and context. During the first phase of this research, a qualitative exploratory study was performed on 11 top executives from the steelmaking industry, the analysis of this information reveals that human error is present in strategic decision-making process.
\end{abstract}

Key words: human error, strategic decision-making, top management.

JEL: M00, M10.

Resumen. La toma de decisiones estratégicas es fundamental en las empresas para mantener sus ventajas competitivas y así asegurar su permanencia en el futuro. El propósito del presente trabajo fue identificar los factores del error humano en la toma de decisiones estratégicas en los niveles directivos de la industria siderúrgica mexicana que inciden en los sesgos de dicho proceso. Dentro de los factores, se determinaron entre otros la emocionalidad, la complejidad cognitiva, el tiempo y el contexto. Durante la primera fase de esta investigación se llevó a cabo un estudio exploratorio cualitativo a 11 ejecutivos de primer nivel dentro de la industria acerera, el análisis de esta información pone en evidencia que el error humano está presente en la toma de decisiones estratégicas.

Palabras clave: error humano, niveles directivos y toma de decisiones estratégicas. 


\section{Introducción}

El presente trabajo está enmarcado en el estudio de los factores del error humano en la toma de decisiones estratégicas en los niveles directivos de la industria siderúrgica mexicana. Esta investigación en proceso tiene una orientación cuantitativa, para lo cual se ha llevado a cabo una revisión de literatura académica relacionada con el fenómeno a estudiar y los factores que influyen en el mismo. A lo largo de este trabajo, se presentan aspectos teóricos que forman parte de dicha revisión, que han permitido identificar variables, quedando por razones de espacio, fuera otros elementos teóricos que han formado parte de la literatura recopilada y que complementan la identificación mencionada.

Por otra parte, queriendo profundizar en el contexto, se llevó a cabo un estudio exploratorio cualitativo sobre el tema de la investigación, a un grupo de 11 ejecutivos de la industria acerera, que ha permitido incorporar algunas variables al modelo. Aunque no forma parte del alcance de esta presentación, es importante mencionar que también fueron efectuados estudios de campo a cerca de 85 ejecutivos de primer nivel de las principales acereras del país, cuyos resultados de manera preliminar, nos arrojan indicios de una relación positiva con el fenómeno a estudiar.

Por lo tanto, el objetivo del estudio, utilizando la información de las fuentes señaladas anteriormente, consiste en identificar las variables que permiten establecer un modelo de causa efecto y las hipótesis que corresponden a las relaciones que dicho modelo plantea. Así, en este documento se presentan el planteamiento del problema, los factores que influyen en el fenómeno, las hipótesis específicas, el modelo gráfico propuesto, su objetivo, la pregunta de investigación, la justificación del estudio, el marco teórico, el método y los resultados del estudio exploratorio.

\section{Planteamiento del problema}

\section{Contexto del fenómeno a estudiar}

El concepto error humano en el desarrollo del presente trabajo, será el término genérico utilizado para englobar todas aquellas ocasiones en las que una secuencia planificada de actividades mentales no logra alcanzar el resultado deseado y que no es atribuido al azar (Reason, 1990). 
Se ubica el error humano como una condición latente, que contiene importantes aspectos que contribuyen a ser expresados en algunas ocasiones como causa y motivo en que el directivo de las empresas está propenso precisamente por su condición humana, a cometerlo al momento de tomar decisiones estratégicas, lo cual termina en la generación de situaciones comprometedoras y conflictivas en contextos de alto riesgo y costo, con un deterioro para la empresa.

En cuanto al concepto de toma de decisiones estratégicas se refiere a aquellas decisiones de gran trascendencia, que comprometen una cantidad sustancial de recursos y determinan el futuro de la compañía en el mediano y largo plazo ya que fijan los objetivos y líneas de acción a seguir.

Existen algunos estudios que nos muestran un perfil del directivo mexicano y nos ayudan a comprender la forma en que toman decisiones. Serralde (1987) en las conclusiones de su investigación destaca que el directivo mexicano en la toma de decisiones es compulsivo cuando se trata de la implementación, ignorando que cada hecho necesita un tiempo para su consumación; confiable y respetable, responde ante la superioridad con inusual disciplina, siendo vista por los altos directivos como una persona de empuje, regularmente cumplidora. En situaciones de apremio se torna errático y poco efectivo. Torpe en el trabajo participativo, trata de influir constantemente, arrollando a veces con actitudes prepotentes, a quienes mira hacia abajo. Menciona también, que en las organizaciones mexicanas sólo el alto mando toma decisiones, ya sean estratégicas, operativas o administrativas, y en muy contadas excepciones el segundo nivel de comando está investido de la autoridad para decidir sin el concurso de la superioridad. El papel del gerente mexicano queda reducido a la implantación de decisiones tomadas por el alto mando y en buena medida lo que distingue a la calidad de los gerentes, es su eficacia en la implementación, ya que esto representa bienestar para la superioridad. Irónicamente existe el clamor en el alto mando de que la gerencia no decide y por eso se ve precisado a intervenir en los niveles inferiores de decisión.

En contraparte, Llano (1994) establece como perfil del directivo mexicano un estilo de mando de gran confianza en la autoridad personal y única, el cual considera importantes las opiniones de sus subordinados, juzga que hay motivaciones más importantes que el dinero y piensa que en el jefe valen otras cualidades además de poseer conocimientos. En cuanto a su estilo de estrategia prefiere una estructura plana, no desea la operación directa sino el mando para que otros operen, utiliza como instrumento de control el estado de pérdidas y 
ganancias antes que el balance y es partidario de cambios graduales por encima de los cambios bruscos. En resumen identifica un perfil de directivo más individualista o competitivo, menos cooperativo con sus colegas, y poco inclinado a la delegación del poder a sus subordinados; pero con relevante confianza en las decisiones de grupo y en el peso convincente de la razón.

Un estudio realizado por la Universidad Autónoma Metropolitana (Ramírez, 2014), identifica el perfil del director mexicano como un hombre equilibrado entre el individualismo y la participación en equipo, para el cual la estructura de autoridad es crucial en una organización y la unidad de mando sumamente importante, con deseos de retener el poder. Es partidario de poseer la autoridad única, pero desde esa posición legal asegurada, requiere del parecer de los demás en la toma de decisiones, porque no desea que éstas sean personales, y su mando no resulte individualista.

Los errores en la toma de decisiones estratégicas en las empresas, son cada vez más evidentes y costosos ante un mercado cada día más competitivo, que no permite mucho margen de maniobra y deja fuera de competencia a todas aquellas organizaciones que no aciertan en sus decisiones. De acuerdo a Nutt (2002) más de la mitad de todas las decisiones de negocios fracasan y cerca del $70 \%$ de los esfuerzos de gestión de cambio no tienen éxito (Nutt, 1993, 2002).

En el contexto del presente estudio que es la Industria Siderúrgica, ésta también se ve inmersa en la estadística y por citar algunos ejemplos de errores en la toma de decisiones estratégicas que concluyeron con el cierre o venta de las empresas tenemos en Estados Unidos: Bethlehem Steel Corp. (13,000 trabajadores) (Reutter, 2004; Warren, 2008), LTV Corp. (18,000 trabajadores) (Hoerr, 1988), Wheeling Pittsburgh Steel Corp. (4,800 trabajadores) (Metzgar, 1987), en Canadá: Stelco (2,000 trabajadores) (Hall, 1997), Sydney Steel Corp. (2,460 trabajadores), en Reino Unido: British Steel (268,500 trabajadores) (International Iron and Steel Institute IISI).

En la Industria Siderúrgica Mexicana tenemos el caso de Fundidora de Fierro y Acero de Monterrey, la cual desapareció en Mayo de 1986 (CANACERO) (Novoa, 1989).

En resumen, la correcta toma de decisiones estratégicas en una empresa perteneciente a la industria siderúrgica hoy en día es una de las más importantes y difíciles tareas, ya que con la efectividad y oportunidad con que se tomen dependerá el avance, crecimiento y permanencia en el mercado de la empresa. 


\section{Pregunta de investigación}

Ante la evidente pérdida económica y altos costos, que en el sector siderúrgico nacional se presentan (INEGI, 2014) por variados aspectos debidos al error humano en la toma de decisiones estratégicas, se requiere incrementar el interés de los profesionales en los niveles directivos, mediante la activación de sus procesos psicológicos de las personas asociadas con este concepto; así también surge la necesidad de profundizar en el campo de investigación para generar teoría y conocimiento sobre el tema del error humano en el campo empresarial.

Por lo anterior se establece la pregunta: ¿Está relacionado el error humano en la toma de decisiones estratégicas con el conocimiento, las reglas, la emocionalidad, los descuidos, el contexto, el tiempo y la complejidad cognitiva de los niveles directivos dentro de la Industria Siderúrgica Mexicana?.

\section{Objetivo}

El objetivo general de la investigación es el de identificar si el conocimiento, las reglas, la emocionalidad, los descuidos, el contexto, el tiempo y la diversidad cognitiva están relacionados con el error humano en la toma de decisiones estratégicas en los niveles directivos en la Industria Siderúrgica Mexicana y con base en lo anterior poder determinar los mecanismos de control que permitan prevenir y/o atenuar el impacto que generan en la estabilidad operativa de la empresa.

\section{Proposiciones de la investigación}

Con base en la revisión de reportes científicos recientes sobre diversos factores y su incidencia en el fenómeno, se ha decido investigar sobre estas siete variables directamente relacionadas con el error humano, algunas de las cuales han sido poco estudiadas por lo que nos ofrecen la posibilidad de poder hacer una aportación al conocimiento en la materia.

Derivado de los factores antes enunciados se desarrollaron las siguientes Proposiciones de la Investigación:

P1 - El conocimiento se relaciona de manera positiva con el error humano para la toma de decisiones estratégicas en los niveles directivos.

P2 - Las reglas inciden de manera positiva con el error humano para la toma de decisiones estratégicas en los niveles directivos. 
P3 - La emocionalidad influye de manera positiva con el error humano para la toma de decisiones estratégicas en los niveles directivos.

P4 - Los descuidos impactan de manera positiva con el error humano para la toma de decisiones estratégicas en los niveles directivos.

P5 - El contexto incide de manera positiva con el error humano para la toma de decisiones estratégicas en los niveles directivos.

P6 - El tiempo influye de manera positiva con el error humano para la toma de decisiones estratégicas en los niveles directivos.

P7 - La complejidad cognitiva se relaciona de manera positiva con el error humano para la toma de decisiones estratégicas en los niveles directivos. (Ver Figura1).

\section{Modelo gráfico propuesto}

Figura 1. Modelo gráfico propuesto causa-efecto

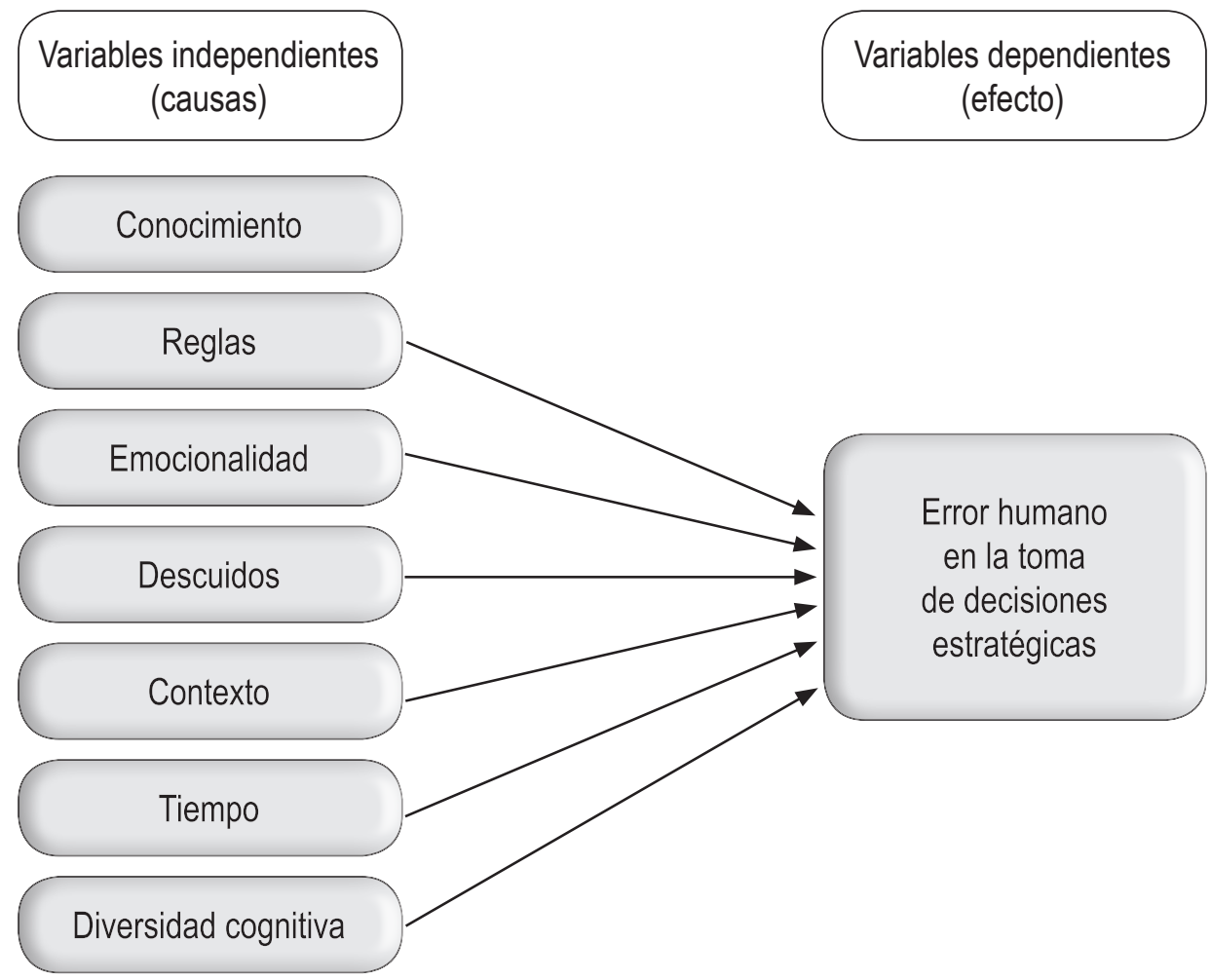

Fuente: Elaboración propia. 


\section{Justificación de la investigación}

La revisión de la información actual sobre la toma de decisiones estratégicas nos indica que entre el $70 \%$ y el $80 \%$ de las quiebras de las empresas y los accidentes laborales pueden ser atribuidos, como mínimo en forma parcial, al error humano (Wickens \& Hollands, 1999). No obstante, atribuirlos únicamente al error de las habilidades técnicas cognitivas de las personas no es suficiente y adicionalmente se deberán investigar las causas específicas.

Hoy en día se tiene claro que dichas quiebras y accidentes no pueden ser atribuidos a una sola causa o, en la mayoría de los casos, incluso a una sola persona. Hasta la identificación de una "causa principal o causa básica" está llena de problemas. Más bien estas quiebras y accidentes constituyen el resultado de varias causas (Bird, 1974; Heinrich, Petersen, \& Ross, 1980; Reason, 1990; Shapell \& Wiegmann, 1997).

El reto y la justificación que se plantea la presente investigación es identificar, delimitar y proponer modelos de acción que permitan mitigar de la mejor manera la secuencia causal de eventos, en particular dicho valor del $70 \%$ y $80 \%$ asociado al error humano ligado a la toma de decisiones estratégica de los niveles directivos en la Industria Siderúrgica Mexicana.

\section{Revisión de literatura}

\section{El fenómeno a estudiar}

El error humano está presente como una situación latente en la diaria toma de decisiones, que tiene una característica intrínseca dentro de la organización, presentando rasgos precisos que nos permiten afirmar que cerca del $70 \%$ de los incidentes ocurren por actos subestándares, es decir, al error humano como es mencionado por Shappell y Wiegmann (Shapell \& Wiegmann, 1996). En Estados Unidos, el Instituto de Normatividad y Tecnología (NIST) reveló que el $70 \%$ de los accidentes y mala toma de decisiones que ocurren dentro del gobierno y en organizaciones privadas son el resultado de errores humanos.

La toma de decisiones estratégicas en la empresa se entiende como una elección que es importante, en términos de las acciones adoptadas, los recursos comprometidos o los precedentes establecidos. Constituye, por consiguiente, un proceso de elección que involucra la asignación de recursos necesarios para 
alcanzar o mantener una ventaja competitiva (Rodríguez, 2007). En este sentido, la toma de decisiones estratégicas es una tarea esencial para la alta dirección, ya que permite a la organización alinear sus recursos y capacidades con las amenazas y las oportunidades que existen en el medio ambiente (Hitt \& Collins, 2007).

Por tanto, las decisiones estratégicas significan elecciones cuyo impacto es de largo alcance. Estas elecciones involucran el empleo de recursos significativos como se mencionó, así como la participación de los más altos niveles y funciones dentro de la empresa (Pedraja \& Rodríguez, 2008). Se ha comprobado empíricamente que casi el 50\% de las decisiones fracasan (Nutt, 2000, 2006, 2008).

El fenómeno a estudiar que se plantea en el presente estudio es el error humano en la toma de decisiones estratégicas, a investigarse en el ámbito de los niveles directivos en la Industria Siderúrgica Mexicana, afiliadas a la CANACERO y con operaciones productivas de Alto Horno en sus plantas, seleccionando para este fin una muestra representativa de aquellos que hayan tomado decisiones estratégicas por más de $\$ 10$ millones de dólares. Por lo anterior, se define el fenómeno de estudio como los fallos en los que una secuencia planificada de actividades mentales tendientes a la toma de decisiones estratégicas no logra alcanzar el resultado deseado y no es atribuido al azar (Reason, 1990).

\section{Los factores que influyen en el fenómeno}

Para el presente estudio se ha determinado considerar como factores que influyen en el fenómeno las siguientes variables independientes que definimos brevemente:

1. El conocimiento, entendido como errores humanos cometidos durante situaciones no familiares para los cuales no existe un "Know How" o guías de control disponibles de casos previos (Dörner \& Schaub, 1994; Mitchell, Shepherd, \& Sharfman, 2011; Rasmussen J., 1983; Sharit, 2012).

2. Las reglas, entendiéndolas como el conjunto de instrucciones o patrones que permiten al ejecutivo tomar decisiones a situaciones para las cuales se poseen ya patrones de soluciones almacenados (Gary, Wood, \& Pillinger, 2012; Rasmussen J., 1983; Reason, 1990).

3. La emocionalidad, la cual se define como la acción del emocional de tomar decisiones, con base en un sentimiento (alegría, placer, tristeza, dolor, etc.) minimizando o pasando por alto indicadores cuantitativos y/o cualitativos 
que bajo otras circunstancias su decisión sería diferente (Contreras \& Aguilera, 2007; Damasio, Damasio, \& Bechara, 2003; Fenton, Soane, Nicholson, \& William, 2011; Jiménez, 2014).

4. Los descuidos, los cuales se definen como las omisiones, negligencias, falta de cuidado y desatenciones en el proceso de la toma de decisiones estratégicas con base en el sesgo cognitivo de exceso de confianza (Cortada, 2008; Del Missier, Mantyla, \& De Bruin, 2012).

5. El contexto, entendiéndolo como el conjunto de circunstancias externas que condicionan un hecho y que afectan al directivo para su toma de decisiones (Shepherd \& Rudd, 2014; Mitchell, Shepherd, \& Sharfman, 2011).

6. El tiempo, considerando el lapso que el ejecutivo toma para el inicio y finalización del proceso de la toma de decisiones (Teichert, Ferrera, \& Grinband, 2014) (Hwang, 1994). El inicio implica el diagnóstico del problema, búsqueda y análisis de alternativas de solución, para finalizar con la selección de la alternativa más conveniente (Kepner \& Tregoe, 1981).

7. La complejidad cognitiva, es una variable que define la complejidad estructural del sistema cognitivo de un individuo, entendiéndose esta como "el grado en que una persona posee la capacidad de percibir el comportamiento de una manera multidimensional" (Schneier, 1979) (Nooraie, 2012).

\section{Marco teórico}

En la revisión efectuada a la literatura y estudios existentes sobre el fenómeno a estudiar y que servirá de base para la presente investigación, se pueden destacar como los más importantes a los siguientes autores en lo relativo al error humano: James Sully con la Teoría de las llusiones (Sully, 1881); William James y Joseph Jastrow y los conceptos sobre la costumbre y lapsus de conciencia (James, 1890; Jastrow, 1905); Sigmund Freud en particular con su Teoría del "Lapsus freudiano" (Freud, 1922); Jens Rasmussen en específico su Modelo SRK de Habilidad Regla - Conocimientos (Rasmussen, 1982); James Reason también sobre su Modelo de Causalidades o Queso Suizo (Reason, 1990) y Nassim Taleb con la Teoría del impacto de lo altamente improbable (Taleb, 2007).

Por la parte de la literatura e investigaciones en materia de la toma de decisiones estratégicas hemos determinado como fundamentales a los siguientes autores: Stephen Ross (1973) y Michael Jensen y William Meckiling (1976) con relación a su Teoría de la Agencia (Jensen \& Meckling, 1976; Ross, 1973), Scott 
Plous en la parte de la psicología del juicio y toma de decisiones (Plous, 1993); William Boulding y su investigación sobre el modelo conceptual de la toma de decisiones en los niveles directivos (Boulding, 1994); Dan Ariely sobre su Teoría de la emocionalidad en la toma de decisiones así como en los conceptos de lo predeciblemente irracional en la toma de decisiones estratégicas (Ariely, 2008); Mark Fenton, Emma Soane, Niegel Nicholson y Paul Willman en relación al papel de las emociones en el proceso de decisiones (Fenton, Soane, Nicholson, \& WiIliam, 2011); Robert Michell, Dean Shepherd y Mark Sharfman que investigaron sobre cuándo y por qué los ejecutivos son inconsistentes en la toma de decisiones estratégicas (Mitchell, Shepherd, \& Sharfman, 2011); Daniel Kahneman sobre su trabajo en psicología y su modelo racional de la toma de decisiones, el impacto de la aversión a la pérdida, el exceso de confianza en las estrategias empresariales y los sesgos cognitivos en estos procesos (Kahneman, 2012) y David Redish abarcando la parte de la neurociencia y neuroeconomía de las decisiones (Redish, 2013).

Respecto a los trabajos de investigación en el campo de la neurociencia podemos citar dos trabajos que se destacan. El primero, el trabajo realizado por Knutson, Rick, Wimmer, Prelec y Lowenstein (2007) que parten del supuesto microeconómico de que los agentes económicos toman sus decisiones sobre la base de sus preferencias y precios de mercado, y utilizando imágenes de resonancia magnética funcional (fMRI) investigan como las personas evalúan sus decisiones y como las distintas partes del cerebro se activan ante perspectivas de ganancia o perdida. Los investigadores obtuvieron resultados significativos en las regresiones estimadas luego de someter a 19 personas a un experimento de toma de decisiones.

El segundo trabajo, el de Kuhnen y Knutson (2005), en el que analizan las desviaciones de la racionalidad que tienen lugar al tomarse decisiones financieras. Utilizando también fMRI, analizan si las anticipaciones de la actividad neurológica pueden predecir decisiones financieras óptimas o sub óptimas. La activación del núcleo accumbens precede a elecciones menos riesgosas, mientras que la activación de la ínsula precede a anticipación de perdidas. La excesiva activación de esos circuitos puede llevar a errores en las decisiones relacionadas con estratégicas. 


\section{Estudio exploratorio cualitativo. Metodología de análisis}

Para conocer la manera de tomar decisiones estratégicas y la percepción de las razones de las equivocaciones en la toma de decisiones, se realizó un sondeo de opiniones a un grupo de 11 ejecutivos de la industria acerera. Posteriormente, se llevó a cabo un análisis de contenido de las respuestas de los entrevistados. En este sentido, la orientación de este apartado es cualitativa. Lo que se busca es mostrar características de la toma de decisiones y la percepción de los aspectos que influyen en la toma de decisiones equivocada, de acuerdo a lo señalado por ellos mismos.

\section{Decisiones que toman}

La primera pregunta estuvo relacionada con las decisiones estratégicas que cada uno de ellos toma. Un primer aspecto que destaca al ser señalado por varios de ellos, es el entorno que en estos últimos tiempos está viviendo el mercado del acero, con precios a la baja y con una limitación de recursos, situación que genera que se estén adecuando las decisiones a los cambios que se les están presentando.

Vale la pena señalar, también, que algunas de las decisiones mencionadas dependen de la función o puesto que desempeñan. Además, existe la posibilidad de que algunas de dichas decisiones puedan tener una influencia determinante sobre los resultados y cumplimiento de las metas que tienen establecidas.

Entre las decisiones tomadas encontramos los siguientes:

1. Definición y selección de alternativas de solución específicas a necesidades de diseño, logística e inversión. 2. Planes y programas para las actividades de ejecución y prueba de nuevos equipos. 3. Decisiones de proyectos de inversión, de venta o desincorporación de una empresa y de compra o incorporación de una nueva compañía.

Entre los criterios usados al tomar la decisión se mencionaron los siguientes:

1. Discriminando los requerimientos de inversión entre diferentes opciones. 2. Es importante que se seleccione una tecnología ya probada y que incluya la capacitación correspondiente tanto para el personal que operará los equipos como al personal de mantenimiento. 3. Los equipos que se ofrezcan y que se seleccionen deberán ser de última tecnología (state of the art) asegurando y garantizando que tanto la capacidad de producción como de calidad se vayan a dar. 
Entre los beneficios/ahorros de tomar la decisión se mencionaron los siguientes:

1. Nuevos equipos que originan sinergias operativas y aportarían mayor volumen de producción/venta. 2. Para satisfacer el mercado en el que se está posicionado. 3. Decisión de comprar en vez de producir, dadas las condiciones del mercado.

La respuesta de estos ejecutivos a la pregunta permite encontrar diferentes tipos de decisiones que podemos inferir corresponden a su función y área de influencia, por lo tanto, están influidas por las mismas. También, en sus respuestas encontramos algunos de los criterios que toman en cuenta en el proceso de tomar la decisión. De igual manera, mencionaron beneficios y ahorros que la decisión puede producir, lo que permite mejorar el desempeño de las organizaciones a las que pertenecen.

De acuerdo a la información revisada con anterioridad se presentan como conclusiones de esta ponencia, diversos aspectos que dan estructura y solidez a la investigación cuantitativa que se está desarrollando, tal como se señaló con anterioridad. Dentro de los hallazgos que encontramos en el estudio exploratorio podemos destacar los siguientes apartados.

\section{Elementos que influyen en una toma de decisiones errónea}

En cuanto a las razones o elementos que influyen en una equivocada toma de decisiones estratégicas, las cuales se mencionaron en varias preguntas, se identificaron similitudes en el análisis de contenido, dichas similitudes permiten establecer algunas categorías, es necesario señalar que algunas diferencias relevantes que se encontraron, también se incorporan al análisis, así, primero, se encuentran las similitudes y al final las diferencias.

\section{Categorías en base a similitudes}

- Información incorrecta o falta de información.

- Visión, Metas y Objetivos, Planeación, Fortalezas.

- Opiniones encontradas.

- La participación de la alta dirección.

- Análisis incorrecto.

- Cambios en la ejecución del proyecto. 
- Urgencia o precipitación en la toma de decisiones.

- Bajo nivel emocional y racional.

- Disponibilidad de recursos.

- El entorno.

- El factor humano.

\section{Diferencias relevantes en lo mencionado}

- Alto coeficiente emocional.

- Experiencia.

- Mala asesoría externa.

- Barreras.

- La intuición.

\section{Método}

Esta investigación tiene una sustentación positivista lógica al establecer como son las cosas, no como deberían ser, es decir, se evita introducir juicios de valor. Así mismo una sustentación filosófica analítica al adoptarse la reflexión racional rigurosa y sistemática sobre las condiciones correctas de la aplicación de conceptos.

Su diseño es no experimental, transeccional, es decir, del tipo descriptivo, correlacional-causal en el año 2016 - 2017 para determinar la relación que existe entre las variables independientes conocimiento, reglas, emociones, descuidos, contexto, tiempo y diversidad cognitiva, con la variable dependiente error humano en la toma de decisiones estratégicas en los niveles directivos de la industria siderúrgica mexicana.

La naturaleza del estudio es de tipo mixto, cualitativo y cuantitativo. Para la parte cualitativa se utilizó la encuesta, la cual nos permite validar las variables utilizadas mediante análisis de contabilidad al ser aplicado a un grupo de directivos seleccionados. El estudio cualitativo será medido con el conteo, el análisis de frecuencia y el análisis estadístico, tanto descriptivo como inferencial de los datos recabados de las variables durante el estudio de campo de la investigación. La población meta del estudio es el conjunto de directivos de las diferentes empresas que conforman la Industria Siderúrgica Mexicana, dentro de la cual se estudiará sólo una parte de la población referida, elegida por cálculo muestral de tal forma que dicha muestra presente indicadores sobre la totalidad de la 
observación de la población. Para determinar el tamaño de la muestra se aplicó la fórmula (1), para el cálculo de intervalo:

donde:

$$
n=\frac{N P Q}{(N-1)\left(\frac{e}{z}\right)^{2}+P Q}
$$

$\mathrm{N}=$ tamaño de la población

$\mathrm{n}=$ tamaño de la muestra

$\mathrm{P}=$ proporción del evento de interés

$\mathrm{Q}=$ complemento de $\mathrm{P}$

e = error tolerado en porcentaje estimado

$\mathrm{z}=$ valor de la distribución normal estandarizada

El cálculo del tamaño de la muestra para variables cuantitativas aleatorias simples de una población finita (2) se obtiene mediante la siguiente ecuación:

$$
n=\frac{N s^{2}}{(N-1)\left(\frac{d}{z}\right)^{2}+s^{2}}
$$

donde:

$n=$ tamaño de la muestra

$N=$ tamaño de la población

$s^{2}=$ desviación estándar (estimada para este estudio)

$d=$ es el error tolerado o distancia de los límites del intervalo en relación a la media muestral expresado en porcentaje (5\%)

$z=$ valor de la distribución normal estandarizada con un intervalo de confianza de $95 \%$

Tabla 1. Varianzas de los datos dependiendo el número de puntos de la escala de Likert

\begin{tabular}{cccc}
\hline $\begin{array}{c}\text { Puntos en la escala } \\
\text { de Likert }\end{array}$ & Media & $\begin{array}{c}\text { Varianza en } \\
\text { distribución normal }\end{array}$ & $\begin{array}{c}\text { Varianza en } \\
\text { distribución uniforme }\end{array}$ \\
\hline 4 & 2.5 & 0.7 & 1.3 \\
5 & 3.0 & 1.2 & 2.0 \\
6 & 4.0 & 2.0 & 3.0 \\
7 & 5.0 & 2.5 & 4.0 \\
10 & 5.5 & 3.0 & 7.0 \\
\hline
\end{tabular}

Fuente. Rositas (2014, p. 251) 
En la ecuación (3), se utilizó la varianza para una distribución uniforme de una escala de Likert de 5 puntos. La varianza tiende a un valor de 2.0 y la media es de 3.0 (Tabla 1). El error tolerado toma un valor de 0.10 considerando un $95 \%$ de confianza, con un valor de población de 90, de la ecuación 2 tenemos:

$$
n=\frac{90(2)}{(89)\left(\frac{0.10}{1.96}\right)^{2}+2}=\frac{180}{4.23}=42.6
$$

$$
n=43
$$

\section{Elaboración de instrumentos de medición}

Se desarrollaron los instrumentos, pruebas y análisis formales de carácter científico necesarios para la obtención de evidencia objetiva suficiente para el sustento de las inferencias que se pretende generar. Los instrumentos se validaron mediante la consulta con académicos y directivos de las empresas siderúrgicas, la confiabilidad se determinó con el coeficiente de a (alfa) de Cronbach; valorado con un nivel de confianza del $95 \%$ utilizando el software Excel, SPSS 21.0 y SmartPLS 3.2.5.

\section{Validez y confiabilidad}

La validez durante la presente investigación, también denominada exactitud, se considera como el grado en que una medición refleja la realidad de un fenómeno o capacidad de medición o instrumento para aquello que fue propuesto, es decir, que mida o clasifique lo que efectivamente analizamos (Manterola, 2002). Se toma en cuenta para la validez:

Validez de Contenido: El grado en el que el instrumento representa la totalidad del fenómeno que se pretende medir (Devlin, Dong, \& Brown, 1993).

Validez de Criterio: Pretende correlacionar las medidas de la escala con otra medición del atributo estudiado (Streiner, Norman, \& Cairney, 2003).

Validez de Constructo: Pretende hacer referencia a la obtención de evidencia empírica que garantice la existencia del constructo en cuestión (Streiner, Norman, \& Cairney, 2003).

En la evaluación de la validez del constructo o concepto existen dos tipos de validez: (a) validez convergente, que muestra en qué medida ítems diseñados para medir un mismo concepto están (co)relacionados entre sí, y (b) validez discriminante la cual muestra en qué medida ítems diseñados para medir concep- 
tos distintos, aunque relacionados, están (co)relacionados entre sí. En la parte de confiabilidad, también denominado precisión, se considera como el grado en que los puntajes de la medición se encuentren libres de error de medida. A través de los coeficientes de correlación de Pearson fue validada la confiabilidad de las encuestas. Se utilizó también dentro de los métodos y técnicas de confiabilidad el coeficiente Alfa de Cronbach, para medir y validar la consistencia de las preguntas de las encuestas como resultado del análisis del cuestionario, ya que este coeficiente nos indica la relación entre los reactivos y la puntuación total del instrumento (Berry \& Feldman, 1985).

Para lo mencionado en los párrafos anteriores, se realizó la validez de contenido con base en una metodología de dos fases, dentro de las cuales se buscó en primer lugar tener una correcta clasificación de los ítems en los constructos previamente definidos y por otra parte evaluar su grado de relevancia (Mendoza \& Garza, 2009). En total fueron seleccionados 7 expertos con base en un perfil previamente definido. Dichos jueces fueron ejecutivos de primer y segundo grado jerárquico en la industria siderúrgica, así como académicos con grado de doctorado con una trayectoria de amplio reconocimiento, involucrados en competencias genéricas como las propuestas en esta investigación, para que existiera concordancia y relevancia entre las evaluaciones de los expertos o jueces, según Kerlinger \& Lee (2002). Con base en los resultados obtenidos, se calculó el índice de Alfa de Cronbach al modelo en su conjunto dando un resultado de confiabilidad del instrumento del 0.789 lo cual es nivel aceptable de fiabilidad (Rositas J., 2014). En la tabla 2 se muestran los índices de las variables independientes del modelo de manera individual.

Tabla 2. Resultados del análisis de confiabilidad de las variables

\begin{tabular}{cc}
\hline Variables & Alfa de Cronbach \\
\hline Conocimiento & 0.709 \\
Emocionalidad & 0.714 \\
Descuidos & 0.704 \\
Reglas & 0.879 \\
Contexto & 0.776 \\
Tiempo & 0.702 \\
Diversidad Cognitiva & 0.707 \\
\hline
\end{tabular}

Fuente. Elaboración propia del autor, utilizando el software SPSS 21.0 


\section{Resultados y conclusiones}

\section{Conclusión del estudio exploratorio}

La reflexión sobre la presencia del error humano en la toma de decisiones estratégicas en las organizaciones nos ha llevado en el terreno práctico a investigar sobre este fenómeno y su incidencia en la industria siderúrgica mexicana, a efecto de poder identificar y validar algunos de los factores más relevantes que inciden en esta problemática. En una primera fase hemos trabajado con un grupo de ejecutivos a través de un par de entrevistas, a fin de sondear sus experiencias en la toma de decisiones estratégicas y tratar de poner en claro, con cuales de los factores del error humano que seleccionamos como variables del modelo se han enfrentado.

Si bien es cierto que la toma de decisiones estratégicas en algunos casos está determinada por el grado de liderazgo de la alta dirección, es más que evidente una vez analizada la información de las respuestas, que el error humano está presente en muchas de ellas y la gran parte de las veces se decidió no considerarlo.

Con base en los resultados del estudio exploratorio cualitativo se adicionaron variables al modelo y se enriqueció el objetivo del estudio.

Dado que la presente es una investigación en proceso aún no están disponibles los resultados y conclusiones finales.

\section{Referencias}

Ariely, D. (2008). Predictably irrational: The hidden forces that shape our decisions. New York, NY: HarperCollins Publishers.

Berry, W., \& Feldman, S. (1985). Multiple regression in practice. USA: SAGE Publications.

Bird, F. E. (1974). Management guide to loss control. Atlanta, GA: Institute Press.

Boulding, W., Moore, M. C., Staelin, R., Corfman, K. P., Dickson, P. R., Fitzsimons, G., Weitz,

B. A. (1994). Understanding managers' strategic decision-making process. Marketing Letters, 5, 413-426.

Contreras, J., \& Aguilera, A. (2007). Emocionalidad y racionalidad en la toma de decisiones conjuntas: Una aproximación modélica con sistemas multiagente. Administración y organizaciones, 49-61.

Cortada, N. (2008). Los sesgos cognitivos en la toma de decisiones. International Journal of Psychological Research, 68-73. 
Damasio, A., Damasio, H., \& Bechara, A. (2003). Emotion, decision making and the orbitofrontal cortex. Oxford Journal, 295-307.

Del Missier, F., Mantyla, T., \& De Bruin, W. (2012). Decision making competence, executive functioning and general cognitive abilities. Journal of Behavioral Decision Making, 331-351.

Devlin, S., Dong, H., \& Brown, M. (1993). Selecting a scale for measuring quality. Marketing research, 12-7.

Dörner, D., \& Schaub, H. (1994). Errors in planning and decision making and the nature of human information processing. Applied Psychology, 433-453.

Fenton-O'Creevy, M., Soane, E., Nicholson, N., \& Willman, P. (2011). Thinking, feeling and deciding: The influence of emotions on the decision making and performance of traders. Journal of Organizational Behavior, 32, 1044-1061.

Freud, S. (1922). Introductory lectures on psychoanalysis. (J. Riviere, Trans.). Londres: George Allen \& Unwin.

Gary, M. S., Wood, R. E., \& Pillinger, T. (2012). Enhancing mental models, analogical transfer, and performance in strategic decision making. Strategic Management Journal, 33, 1229-1246.

Hall, C. L. G. (1997). Steel Phoenix: The fall and rise of the U.S. steel industry. New York, NY: Palgrave Macmillan.

Heinrich, H., Petersen, D., \& Ross, N. (1980). Principles of accident prevention. In industrial accident prevention. New York, NY: McGraw-Hill.

Hitt, M. A., \& Collins, J. D. (2007). Business ethics, strategic decision making, and firm performance. Business Horizons, 50, 353-357.

Hoerr, J. P. (1988). And the wolf finally came: The decline of the American steel industry. Pittsburgh, PA: University of Pittsburgh Press.

Hwang, M. (1994). Decision making under time pressure: A model for information systems research. Information \& Management, 197-203.

INEGI. (2014). La industria Siderúrgica en México 2013. México: Serie Estadísticas Nacionales. James, W. (1890). The principles of psychology. New York, NY: Holt.

Jastrow, J. (1905). The lapses of consciousness. The Popular Science Monthly, 67, 481-502. Jensen, M. C., \& Meckling, W. H. (1976). Theory of the firm: Managerial behavior, agency costs and ownership structure. Journal of Financial Economics, 3, 305-360.

Jiménez, A. (2014). La retórica clásica y la neurociencia actual: Las emociones y la persuasión. Retor, 56-83.

Kahneman, D. (2012). Thinking, fast and slow. New York, NY: Farrar, Straus \& Giroux.

Kepner, C., \& Tregoe, B. (1981). The new rational manager. New Jersey: Princeton Research Press.

Kerlinger, F., \& Lee, H. (2002). Investigación del comportamiento: Métodos de investigación en Ciencias Sociales. México: McGraw-Hill.

Knutson, B., Rick, S., Wimmer, E., Prelec, D., \& Loewenstein, G. (2007). Neural predictors of purchase. Neuron, Elsevier, 147-156. 
Kuhnen, C., \& Knutson, B. (2005). The neural basis of financial risk taking. Neuron, 763-770. Llano, C. (1994). El nuevo empresario en México. México: Fondo de Cultura Económica. Manterola, C. (2002). El proceso de medición con variables cualitativas. Revista Chilena de Cirugía, 307-315.

Mendoza, J., \& Garza, J. (2009). La medición en el proceso de investigación científica: Evaluación de validez de contenido y confiabilidad. InnOvaciOnes de NegOciOs, $17-32$.

Metzgar, J. (1987). Firing the boss! The steelworkers at wheeling-pitt. Labor Research Review, 1, 63-77.

Mitchell, J. R., Shepherd, D. A., \& Sharfman, M. P. (2011). Erratic strategic decisions: When and why managers are inconsistent in strategic decision making. Strategic Management Journal, 32, 683-704.

Nooraie, M. (2012). Factors influencing strategic decision making processes. International Journal of Academic Research in Business and Social Science, 2(7), 405-429.

Novoa, J. Z. (1989). La muerte de fundidora: Reconversión de la cultura industrial mexicana. México D.F.: LIMUSA.

Nutt, P. (1993). Surprising but true: Half the decision in organizations fail. Academy of Management Review, 138(4), 75-90.

Nutt, P. (2000). Decision Making Success in Public, Private and Third Sector Organizations: Finding Sector Dependent Best Practice. Journal of Management Studies, 1(37), 77-109.

Nutt, P. (2002). Why decisions fail: Avoiding the blunders and traps that lead to debacles. San Francisco, CA: Berrett-Koehler Publishers.

Nutt, P. (2006). Comparing public and private sector decision making practices. Journal of Public Administration Research and Theory, 2(16), 289-318.

Nutt, P. (2008). Investigating the success of decision making processes. Journal of management studies, 425-455.

Pedraja, L., \& Rodríguez, E. (2008). Estilos de liderazgo, gestión del conocimiento y diseño de la estrategia: Un estudio empírico en pequeñas y medianas empresas. (Vol. 9). Caracas, Venezuela.

Plous, S. (1993). The psychology of judgment and decision making. New York, NY: McGraw-Hill Education.

Ramírez, F. (2014). El perfil del director mexicano, Universidad Autónoma Metropolitana, en http://uami. wikispaces.com/El+perfil+del+director+mexicano

Rasmussen, J. (1982). Human errors. A taxonomy for describing human malfunction in industrial installations. Journal of Occupational Accidents, 4, 311-333.

Rasmussen, J. (1983). Skills, rules and knowledge: signals, signs and symbols and other distractions in human performance models. IEEE Transactions on systems, man and cybernetics, 257-266.

Reason, J. (1990). Human error. Cambridge, UK: Cambridge University Press.

Redish, A. D. (2013). The mind within the brain. Oxford: Oxford University Press. 
Reutter, M. (2004). Making steel: Sparrows point and the rise and ruin of American industrial might. Chicago: University of Illinois Press.

Rodríguez, E. (2007). La toma de decisiones estratégica en las pequeñas y medianas empresas. Sociedad \& Información, 8(1), 5-8.

Rositas, J. (2014). Los tamaños de las muestras en encuestas de las ciencias sociales y su repercusión en la generación del conocimiento. InnoOvacioOnes de NegOcios, 235-268.

Ross, S. A. (1973). The economic theory of agency: The principal's problem. American Economic Review, 63, 134-139.

Schneier, C. (1979). Measuring cognitive complexity: Developing reliability, validity and norm tables for a personality instrument. Educational and Psychological Measurement, 39(3), 599-612.

Serralde , A. (1987). El estilo mexicano de dirigir. Management Today en Español, enero, $5-20$.

Shapell, S. A., \& Wiegmann, D. A. (1996). US Naval Aviation mishaps 1977-1992: Differences between single and dual piloted aircraft. USA: Aviation, Space and Enviromental Medicine.

Shapell, S. A., \& Wiegmann, D. A. (1997). Human Factors Analysis of Postaccident Data: Applying theoretical taxonomies of human error. The international journal of aviation psychology.

Sharit, J. (2012). Human Error and Human Realiability Analysis. En G. Salvendry, Handbook of Human Factors and Ergonomics, 734-800. New Jersey: John Wiley \& Sons Inc.

Shepherd, N. G., \& Rudd, J. M. (2014). The influence of context on the strategic decisionmaking process: A review of the literature. International Journal of Management Reviews, 16, 340-364.

Streiner, D., Norman, G., \& Cairney, J. (2003). Health measurement scales: A practical guide to their development and use. London: Oxford University Press.

Sully, J. (1881). Illusions: A psychological study. Londres, UK: C. Keagan Paul and Co.

Taleb, N. N. (2007). El cisne negro. Barcelona, España: Editorial Planeta.

Teichert, T., Ferrera, V., \& Grinband, J. (march de 2014). Humans Optimize Decision-Making by delaying decision onset. Plos one, 9(3), 1-22.

Warren, K. (2008). Bethlehem steel: Builder and arsenal of America. Pittsburgh, PA: University of Pittsburgh Press.

Wickens, C. D., \& Hollands, J. G. (1999). Engineering psychology and human performance. New York, NY: Pearson. 